\title{
Glacier Velocity Monitoring by Maximum Likelihood Texture Tracking
}

\author{
Esra Erten, Student Member, IEEE, Andreas Reigber, Member, IEEE, \\ Olaf Hellwich, Senior Member, IEEE, and Pau Prats, Member, IEEE
}

\begin{abstract}
The performance of a tracking algorithm considering remotely sensed data strongly depends on a correct statistical description of the data, i.e., its noise model. The objective of this paper is to introduce a new intensity tracking algorithm for synthetic aperture radar (SAR) data, considering its multiplicative speckle/ noise model. The proposed tracking algorithm is discussed regarding the measurement of glacier velocities. Glacier monitoring exhibits complex spatial and temporal dynamics including snowfall, melting, and ice flows at a variety of spatial and temporal scales. Due to these complex characteristics, most traditional methods based on SAR suffer from speckle decorrelation that results in a low signal-to-noise ratio. The proposed tracking technique improves the accuracy of the classical intensity tracking technique by making use of the temporal speckle structure. Even though a new intensity-based matching algorithm is proposed, particularly for incoherent data sets, the analysis of the proposed technique was also performed for correlated data sets. As it is demonstrated, the velocity monitoring can be continuously performed by using the maximum likelihood (ML) texture tracking without any assumption concerning the correlation of the data set. The ML texture tracking approach was tested on ENVISAT-ASAR data acquired during summer 2004 over the Inyltshik glacier in Kyrgyzstan, representing one of the largest alpine glacier systems of the world. It will be demonstrated that the proposed technique is capable of robustly and precisely detecting the surface velocity field and velocity changes in time.
\end{abstract}

Index Terms-Estimation theory, glacier-motion estimation, maximum likelihood (ML) estimation, offset tracking, speckle, synthetic aperture radar (SAR).

\section{INTRODUCTION}

I $\mathrm{N}$ RECENT years, a special interest in long-term operational glacier monitoring has emerged, mainly due to the relation of glacier recession to climate changes. Although in situ observations with the aim of glacier velocity measurement can be very accurate, in practice, it is not feasible to visit every

Manuscript received March 10, 2008; revised August 26, 2008 and October 27, 2008. Current version published January 28, 2009. This work was supported by the German Academic Exchange Service (DAAD).

E. Erten is with the Department of Computer Vision and Remote Sensing, Technical University of Berlin, 10587 Berlin, Germany, and also with the Microwaves and Radar Institute, German Aerospace Center, 82234 Oberpfaffenhofen, Germany (e-mail:esra.erten@dlr.de).

A. Reigber and P. Prats are with the Microwaves and Radar Institute, German Aerospace Center, 82234 Oberpfaffenhofen, Germany (e-mail: andreas.reigber@dlr.de; pau.prats@dlr.de).

O. Hellwich is with the Department of Computer Vision and Remote Sensing, Technical University of Berlin, 10587 Berlin, Germany (e-mail: hellwich@cs.tu-berlin.de).

Color versions of one or more of the figures in this paper are available online at http://ieeexplore.ieee.org.

Digital Object Identifier 10.1109/TGRS.2008.2009932 glacier area regularly. Remotely sensed data provide a simple possibility for automatic monitoring of entire glacier systems. Optical data provided by photogrammetry or through satellite image systems have limitations in glacier monitoring. Most glacierized areas are characterized by a strong weather phenomenon, like thunderstorms, regular rain, and snowfall, that causes a change in illumination. In addition, finding cloudless optical data of such regions on a regular basis, which is necessary to model glacier flow patterns, is not an easy task in practice. In contrast, the synthetic aperture radar (SAR) data have no limitations regarding weather phenomena or illumination.

Up to now, three main approaches have been used for the estimation of flow velocities: optical flow, commonly applied using optical data, interferometric techniques using SAR data, and image matching for all types of remotely sensed data [1]-[4]. However, all methods possess severe disadvantages. Optical flow methods are based on the assumption that the brightness of a pattern is invariant over time, and the image velocity is computed from spatio-temporal intensity gradients using normalization [5]. However, this technique can only be applied on high-quality optical images, which are not easy to acquire. In the case of SAR, this technique is very sensitive to noise because of the use of numerical differentiation.

Although the potential of differential SAR interferometry (DInSAR) for glacier velocity estimation has been shown in several case studies [6]-[8], its successful application is often limited by phase noise. In addition, the glacier flow is often too fast to be analyzed by means of DInSAR because the displacement rate becomes too large during a typical satellite data reacquisition (e.g., 26 days for Radarsat-1, 35 days for ASAR Envisat, and 46 days for ALOS Palsar). Another limitation regarding differential interferometry is that DInSAR only measures the glacier velocity in the line-of-sight (LOS) direction, and hence, it is not possible to retrieve the full 3-D displacement vector. The assumption that the glacier flow is parallel to the surface is generally accepted with the aim of the retrieval of the full displacement vector [8], which needs the digital elevation model (DEM) of the scene to confine the LOS vector along the gradient of the elevation model. In addition, the use of a combination of an ascending and a descending interferogram overcomes this 3-D problem, but with the cost of an additional data set [7]. The interferometric spectral diversity technique (split-band interferometry) overcomes this problem by supplying the velocity information also in the azimuth direction (perpendicular to the LOS) and was successfully applied with the aim of 3-D motion vector extraction [8], [9]. The method estimates absolute phase-absolute range-by calculating the 
phase differences of the two interferograms generated from bandpass-filtered images [10], [11]. Although the spectral diversity technique is well suited for deformation monitoring, this technique also requires a coherent SAR data set, as in the case of DInSAR applications, and is less accurate than DInSAR, since the accuracy of DInSAR is related to the wavelength, not the bandwidth, as in spectral diversity. In contrast, SAR intensity images are not limited by phase stability problems and can be reliably acquired on a regular basis. This makes intensitybased algorithms appealing for operational monitoring.

Intensity-based motion estimation approaches define the displacement as the shift that yields the best fit between different images in time. However, the most common approach, known as cross-correlation, is not the maximum likelihood (ML) approach for SAR intensity images, as it is the ML solution for optical data with an additive noise model [12] and for complex SAR data with circular Gaussian statistics [13]. Therefore, traditional methods are not optimal for automatic glacier monitoring because of speckle decorrelation. In order to increase the accuracy of the intensity-based matching algorithm, the texture tracking algorithm is redefined in this paper by ML parameter estimation.

This paper is organized as follows. The ML motion estimation function, considering that SAR intensity has multiplicative speckle statistics, is defined for correlated and uncorrelated speckle in Section II. Sections III and IV present the experimental results, including the comparison of accuracy between the proposed and the traditional approaches on simulated and real data. Section $\mathrm{V}$ concludes this paper with discussions and directions for future work.

\section{Problem Formulation AND Architecture of THE PRoposed TECHNIQUES}

The classical intensity matching algorithm finds the local shift between images using a normalized cross-correlation (NCC) algorithm. The best match is found by maximizing a similarity measurement through candidate blocks [2]-[4], [14]. Then, confidence is interpreted by comparing the value of the correlation peak related to that of the mean and the minimum correlation. In this section, this matching algorithm is improved based on the multiplicative noise model of the SAR intensity with the assumption of fully developed speckle.

According to the definition of the ML motion estimation as indicated in [12] and [15], the matching algorithm performance is directly obtained through maximizing the conditional density function (CDF) of two matched blocks.

Supposing that a block $y_{i}=\left[y_{i 1}, \ldots, y_{i k}\right]^{\mathrm{T}}$ matches a block $x_{i}=\left[x_{i 1}, \ldots, x_{i k}\right]^{\mathrm{T}}$, this matching results in a displacement (shift) vector $v_{i}$, where $i$ indicates the block which is shifted $i$ pixels and $k$ is the number of pixels in blocks $y_{i}$ and $x_{i}$. Here, blocks are obtained from two intensity SAR images $Y$ and $X$ of size $M \times N$ acquired over the same area at different times $t_{1}$ and $t_{2}$. Then, the ML estimate $\vec{v}_{\mathrm{ML}}$ of $\vec{v}_{i}$ is obtained by maximizing, for each block $i$, the following CDF function:

$$
\max _{\vec{v}} p\left(x_{i} \mid y_{i}, \vec{v}\right), \quad \vec{v}=\vec{v}_{\mathrm{ML}}
$$

Note that the vectors (blocks) $x_{i}$ and $y_{i}$ consist of uncorrelated pixels ${ }^{1}$ having uniform motion.

ML motion estimation, as defined in (1), will be discussed in the following with emphasis on uncorrelated speckle, but the performance for correlated speckle is also assessed. However, it should be remembered that, for correlated speckle patterns, the coherent cross-correlation technique is a very accurate technique providing the ML estimate for high coherence [13] (the reader is invited to look at [11] and [13] for more details about coherent cross-correlation techniques).

\section{A. Multiplicative Speckle Model With Uncorrelated Speckle Within and Between Images}

SAR intensity $(I)$ images are described by a multiplicative speckle noise with the following probability density function (pdf) [16]:

$$
I=\sigma \eta, \quad p_{\eta}(\eta)=\frac{N^{N} \eta^{N-1}}{\Gamma(N)} \exp (-N \eta)
$$

where $\sigma, N$, and $\eta$ indicate the radar cross section (RCS), i.e., reflectivity of object, number of looks, and speckle, respectively. Under the assumption of a multiplicative speckle model, the RCS and speckle value of the $j$ th pixel in block $i$, at time $t_{1}$, are $\sigma_{i j}^{\prime}$ and $\eta_{i j}^{\prime}$, respectively, and that of at time $t_{2}$ are $\sigma_{i j}^{\prime \prime}$ and $\eta_{i j}^{\prime \prime}$. Then, the models for the observed pixels at time $t_{1}$ and $t_{2}$ are

$$
\begin{aligned}
& y_{i j}=\sigma_{i j}^{\prime} \eta_{i j}^{\prime} \\
& x_{i j}=\sigma_{i j}^{\prime \prime} \eta_{i j}^{\prime \prime} .
\end{aligned}
$$

To maximize the $\mathrm{CDF}$ as defined in (1), the relationship between images is redefined as

$$
x_{i j} \equiv \alpha_{i j} y_{i j} \gamma_{i j} \leftarrow\left\{\begin{array}{l}
\alpha_{i j}=\frac{\eta_{i j}^{\prime \prime}}{\eta_{i j}^{\prime}} \\
\gamma_{i j}=\frac{\sigma_{i j}^{\prime \prime}}{\sigma_{i j}^{\prime}} .
\end{array}\right.
$$

This new multiplicative image-speckle model, which is extracted from the ratio of two speckle variables, has the advantage of being unaffected by multiplicative calibration errors and geometry-based imaging effects on the backscattering coefficients. Regarding its advantages, it is very common to work with such ratio images in the application area of change and edge detection (see, e.g., [16] and [17]). With uncorrelated images, the new speckle $\left(\alpha_{i j}\right)$ has a new distribution calculated from the ratio of two independent variables (independent speckle model of images at $t_{1}$ and $t_{2}$ ). If $\eta^{\prime}$ and $\eta^{\prime \prime}$ are equally distributed independent variables, as it is the case for decorrelated images, the pdf of $\alpha=\eta^{\prime \prime} / \eta^{\prime}$ is given by [15, eq. 6.56]

$$
p_{\alpha}(\alpha)=\int_{0}^{\infty} p_{\eta}\left(\alpha \eta^{\prime}\right) p_{\eta}\left(\eta^{\prime}\right) \eta^{\prime} d \eta^{\prime}, \quad \eta^{\prime}, \eta^{\prime \prime} \geq 0 .
$$

\footnotetext{
${ }^{1}$ In practice, neighboring pixels may have a small amount of correlation based on oversampling (related to widths of the point spread function) of the SAR data. However, in our case, this can be ignored [16, ch. 8]
} 
By substituting (2) in (6), it follows that

$$
\begin{aligned}
p_{\alpha}(\alpha) & =\frac{N^{2 N} \alpha^{N-1}}{\Gamma(N) \Gamma(N)} \int_{0}^{\infty} \eta^{2 N-1} \exp \left(-\eta^{\prime}(N \alpha+1)\right) d \eta^{\prime} \\
& =\frac{\Gamma(2 N)}{\Gamma(N) \Gamma(N)} \frac{\alpha^{N-1}}{(1+\alpha)^{2 N}}
\end{aligned}
$$

where $p_{\alpha}(\alpha)$ indicates the new speckle distribution [Fig. 1(a)]. If the random variables $x_{i}$ and $y_{i}$ are statistically independent, then the conditional probability of $x_{i}$ given $y_{i}$ is $p\left(x_{i} \mid y_{i}\right)=$ $p\left(x_{i}\right)$. Considering (5), $p\left(x_{i}\right)$ can be derived using the change of variable relation

$$
x_{i j}=\alpha_{i j} y_{i j} \gamma_{i j} \rightarrow\left\{p\left(x_{i j}\right)=\frac{p_{\alpha}\left(\alpha_{i j}\right)}{y_{i j} \gamma_{i j}} .\right.
$$

This yields that the maximization of (1) is equivalent to

$$
\begin{aligned}
\max _{\vec{v}_{i}} \prod_{j=1}^{k} \frac{1}{y_{i j} \gamma_{i j}} p_{\alpha}\left(\alpha_{i j}\right) \rightarrow \vec{v}_{i}=\vec{v}_{i_{\mathrm{ML}}} & \\
p\left(x_{i} \mid y_{i}, \vec{v}_{i}\right) & =\prod_{j=1}^{k} \frac{\left(\frac{x_{i j}}{y_{i j} \gamma_{i j}}\right)^{N-1}}{y_{i j} \gamma_{i j}\left(1+\frac{x_{i j}}{y_{i j} \gamma_{i j}}\right)^{2 N}} \frac{\Gamma(2 N)}{\Gamma(N) \Gamma(N)} \\
& =\prod_{j=1}^{k} \frac{\left(\frac{x_{i j}}{y_{i j}}\right)^{N-1} y_{i j}\left(1+\frac{x_{i j}}{y_{i j}}\right)^{2 N} \frac{\Gamma(2 N)}{\Gamma(N)^{2}}, \quad \gamma=1 .}{}
\end{aligned}
$$

From (9), it can be seen that in order to calculate the ML motion estimate, $\gamma=\sigma^{\prime \prime} / \sigma^{\prime}$ should be known. However, in practice, this information is not available. Setting $\gamma=1$ (no change in RCS), which means that two blocks have the same mean and variance, is an option to resolve the missing $\gamma$ information. This condition can be supplied by intensity normalization, which is also common in optical flow and texture analysis techniques. Then, maximizing the CDF is equivalent to maximizing the following objective function:

$m l\left(\vec{v}_{i}\right)=\sum_{j=1}^{k} \ln y_{i j}+\ln x_{i j}-2 \ln \left(x_{i j}+y_{i j}\right)-\frac{\ln x_{i j}}{N}$.

To enhance low-intensity pixels, the ratio images are usually expressed in a logarithmic scale shown by $\underline{X}$ and $\underline{Y}(\log X=$ $\underline{X})$. Then, $m l\left(\vec{v}_{i}\right)$ is redefined for logarithmic images as (see Appendix I)

$$
m l\left(\vec{v}_{i}\right)=\sum_{j=1}^{k} \underline{x_{i j}}-\underline{y_{i j}}-2 \ln \left(1+\exp \left(\underline{x_{i j}}-\underline{y_{i j}}\right)\right) .
$$

In either the maximization of (10) or (11), it is understood that $x_{i j}$ 's are the intensity pixels of block $x_{i}$ obtained from $X$, and $y_{i j}$ 's are the corresponding pixels of block $y_{i}$ obtained from $Y$, if the velocity $\vec{v}_{i}$ is assumed. The objective functions are calculated related to the shifts of a moving block from the first image with respect to the second image. The shift vector having the most likelihood value yields the image offset.

\section{B. Multiplicative Speckle Model With Uncorrelated Speckle Within Images and Correlated Speckle Between Images}

If the speckle is correlated between two images, a bivariate gamma distribution, which takes into account the correlation between variables, may be employed. On the basis of the bivariate gamma distribution introduced in [18], the joint distribution of correlated speckle $\eta^{\prime}$ and $\eta^{\prime \prime}$ characterized by common shape parameter $^{2} 1 / N>0$ is

$$
\begin{aligned}
p_{\eta^{\prime \prime} \eta^{\prime}} & \left(\eta^{\prime \prime}, \eta^{\prime}\right)=\frac{N^{N+1}\left(\eta^{\prime} \eta^{\prime \prime}\right)^{\frac{N-1}{2}}}{\Gamma(N)(1-\rho) \rho^{\frac{N-1}{2}}} \\
& \times \exp \left(-\frac{N\left(\eta^{\prime}+\eta^{\prime \prime}\right)}{1-\rho}\right) I_{N-1}\left(\frac{2 \sqrt{\rho}}{1-\rho} N \sqrt{\eta^{\prime} \eta^{\prime \prime}}\right)
\end{aligned}
$$

where $I_{N-1}$ is the modified Bessel function of the first kind and $0 \leq \rho \leq 1$ is the correlation coefficient [Fig. 1(b)]. It is obvious that, when $\rho \rightarrow 0$, the joint pdf (12) reduces to the product of two univariate gamma distributions. Here, it is worth noting that the aim is to find an ML motion function in the case of correlated speckle without the need of estimating $\rho$. The way to estimate $\rho$ in its ML sense is to derive the ML estimator from (12) [19].

The pdf of the ratio of two correlated speckle distributions is recalculated (see Appendix II); then, the expansion of (7) for correlated speckle is given by

$$
\begin{aligned}
p_{\alpha}(\alpha)=\frac{\Gamma(2 N)}{\Gamma(N) \Gamma(N)}(1 & -\rho)^{N} \frac{\alpha^{N-1}}{(1+\alpha)^{2 N}} \\
& \times{ }_{1} F_{0}\left(N+\frac{1}{2} ;-; \frac{4 \rho \alpha}{(\alpha+1)^{2}}\right) .
\end{aligned}
$$

Fig. 1(c) shows the new speckle distribution calculated from the ratio of two correlated speckle variables with correlation parameter $\rho=0.8$. The ML motion estimation, as defined in (9) for uncorrelated speckle, is extended for correlated speckle by replacing ${ }_{1} F_{0}(a ;-; x)$ with $(1-x)^{-a}$

$$
\begin{aligned}
p\left(x_{i} \mid y_{i}, \vec{v}_{i}\right)= & \prod_{i=1}^{k} \frac{\alpha_{i j}^{N-1}}{\left(1+\alpha_{i j}\right)^{2 N}} \frac{\left(1-\rho_{i j}\right)^{N}}{y_{i j}} \\
& \times{ }_{1} F_{0}\left(N+\frac{1}{2} ;-; \frac{4 \rho_{i j} \alpha_{i j}}{\left(\alpha_{i j}+1\right)^{2}}\right) \\
= & \prod_{i=1}^{k} \frac{\frac{x_{i j}}{y_{i j}}{ }^{N-1}}{\left(1+\frac{x_{i j}}{y_{i j}}\right)^{2 N} \frac{\left(1-\rho_{i j}\right)^{N}}{y_{i j}}} \\
& \times\left(1-\frac{4 \rho_{i j} x_{i j}}{y_{i j}\left(1+\frac{x_{i j}}{y_{i j}}\right)^{2}}\right)^{-\left(N+\frac{1}{2}\right)}
\end{aligned}
$$

After removing the constant terms, the log-likelihood objective function for correlated and logarithmically scaled correlated images can be seen in (15) and (16).

\footnotetext{
${ }^{2}$ Generally, the same region in two different images has the same equivalent number of looks if the imaging geometry has not changed. The assumption of common shape parameter $1 / N$ is hence satisfied.
} 


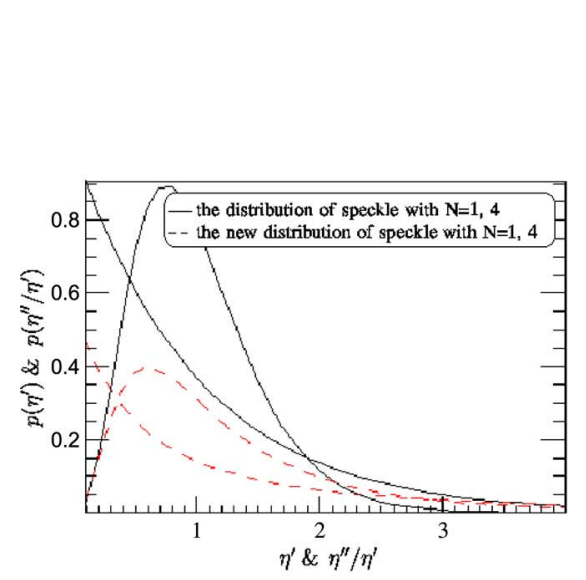

(a)

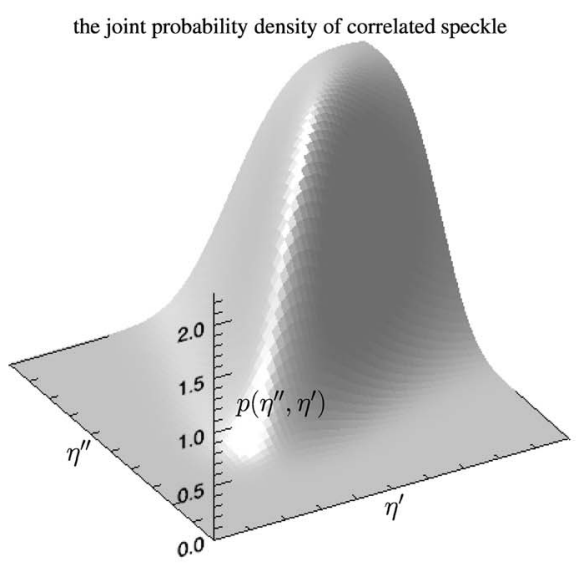

(b)

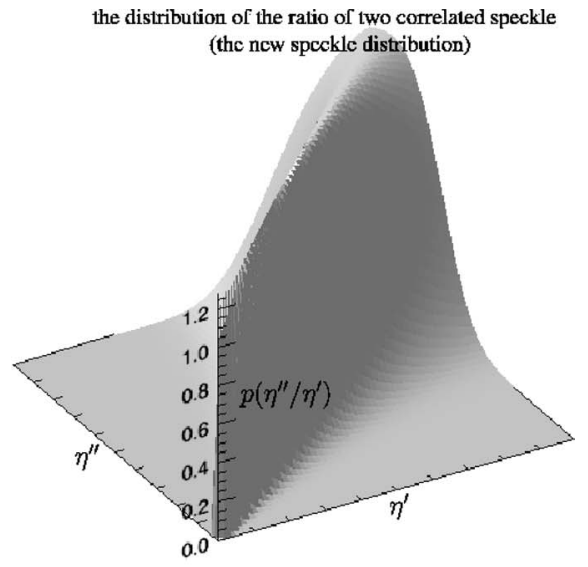

(c)

Fig. 1. (a) Typical speckle distribution and the distribution of the ratio of two uncorrelated speckle variables $\eta^{\prime}$ and $\eta^{\prime \prime}$. (b) Joint pdf of correlated speckle with $N=4$ and $\rho=0.8$. (c) Distribution of the ratio of two correlated speckle variables $\eta^{\prime}$ and $\eta^{\prime \prime}$ in presence of correlation $\rho=0.8$, with $N=4$.

The ML motion vector can be estimated directly from (10), (11), (15), or (16). In whichever maximization (10), (11), (15), or (16), the likelihood value $m l\left(\vec{v}_{i}\right)$ indicates the quality of the matching between block $x_{i}$ and block $y_{i}$, where blocks are constructed related to the shift vector $\vec{v}_{i}$. Then, the motion vector having the most likely value is chosen as a displacement vector.

In estimation theory, the relative entropy is used to measure the difference between two pdfs. In [19], this divergence test is applied between the joint bigamma distribution and the product of two marginal gamma distributions in order to find a threshold for $\rho$ and to make an assumption about the independence of the data. It can be seen from [19] that data with correlation below $\rho=0.5$ may be considered as uncorrelated, and therefore, (10) and (11) should be used instead of

$$
\begin{aligned}
m l\left(\vec{v}_{i}\right)= & \sum_{j=1}^{k} \ln y_{i j}+\left(1-\frac{1}{N}\right) \ln x_{i j}-2 \ln \left(x_{i j}+y_{i j}\right) \\
& -\left(1+\frac{1}{2 N}\right) \ln \left(1-\frac{4 \rho_{i j} x_{i j} y_{i j}}{\left(x_{i j}+y_{i j}\right)^{2}}\right) \\
m l\left(\vec{v}_{i}\right)= & \sum_{j=1}^{k} \underline{x_{i j}}-\underline{y_{i j}}-2 \ln \left(1+\exp \left(\underline{x_{i j}}-\underline{y_{i j}}\right)\right) \\
& -\left(1+\frac{1}{2 N}\right) \ln \left(1-\frac{4 \rho_{i j} \exp \left(\underline{x_{i j}}-\underline{y_{i j}}\right)}{\left(1+\exp \left(\underline{x_{i j}}-\underline{y_{i j}}\right)\right)^{2}}\right) .
\end{aligned}
$$

\section{Results With Simulated Data}

Monte Carlo simulations have been performed to examine the reliability and variability of the proposed and of the classical shift estimator by calculating the percentage of mismatch. For the sake of simplicity, 1-D normally distributed signals with zero mean and a standard deviation of one have been constructed 1000 times. First, each signal has been modified twice by independent zero-mean Gaussian additive noises (to simulate optical images characterized by additive noise structure). Then, the NCC and the proposed ML techniques have been applied to every modified signal pairs with the aim of finding an optimum shift vector. For modified signal pairs, the percentages of correct motion estimation obtained by NCC and ML are $83 \%$ and $54 \%$, respectively. Second, each noise-free signal has been modified twice by independent multiplicative speckle patterns characterized by gamma distribution. For these multiplicative speckle structured signal pairs, the percentages of correct motion estimation obtained by NCC and ML are $68 \%$ and $94 \%$, respectively. Not only the percentage of correct estimations is higher but also the distribution of the ML estimator is almost the same for all simulated signals with a sharp peak at $\vec{v}_{i}=0$. According to the simulated examples, an interesting result is that NCC supplies a very good estimation (1\% mismatch) if only one signal is characterized by additive noise, and the ML function will outperform the NCC function over almost all signals if signals are described by a multiplicative speckle model. The ML estimation with two different objective functions (10)-(15) was also implemented in order to evaluate their performances. Even though small variations in ML values are observed, the location of the ML value through shift vectors is the same for both functions. Fig. 2(a) shows one of the 1-D signals with its additive and multiplicative noise components, and Fig. 2(b) shows the corresponding NCC and ML estimation of the displacement vector through the candidate displacement positions.

\section{Results With ExPerimental Data}

The ML texture tracking approach was tested on ENVISATASAR images acquired on the 19th of August, 23rd of September, and 28th of October 2004 over the Inyltshik glacier in Kyrgyzstan, representing one of the largest alpine glacier systems of the world. Table I summarizes the acquisitions including their system parameters. It is worth mentioning that the precise geometric coregistration of SAR data sets is a strict requirement for matching algorithms. In order to perform a precise coregistration, backward geocoding was employed. In the backward geocoding approach, for each DEM element, the image pixel with the nearest range-Doppler coordinate is calculated [20]. The objective function of (11) was used for the experimental results related to our decorrelated image sequence. However, for operational monitoring applications, the objective function of (16), which also considers correlated 

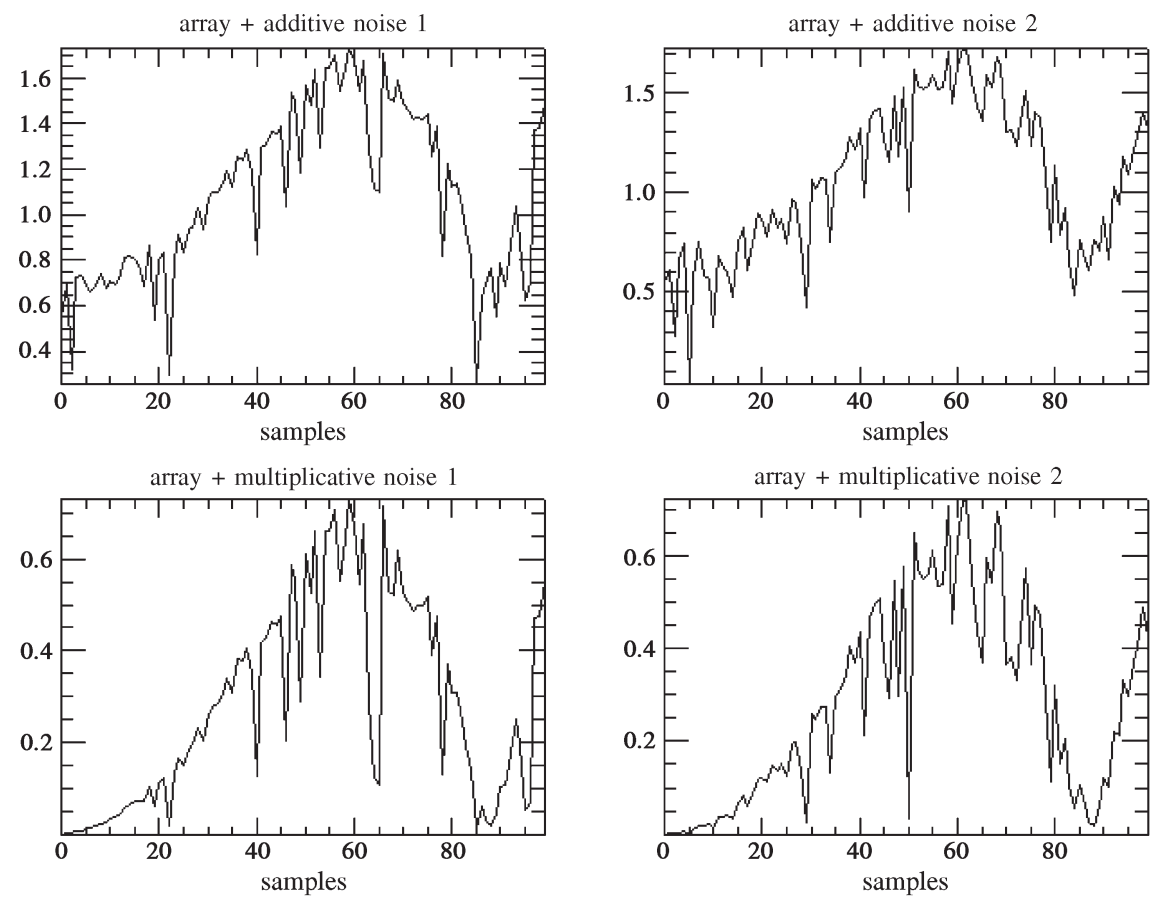

(a)
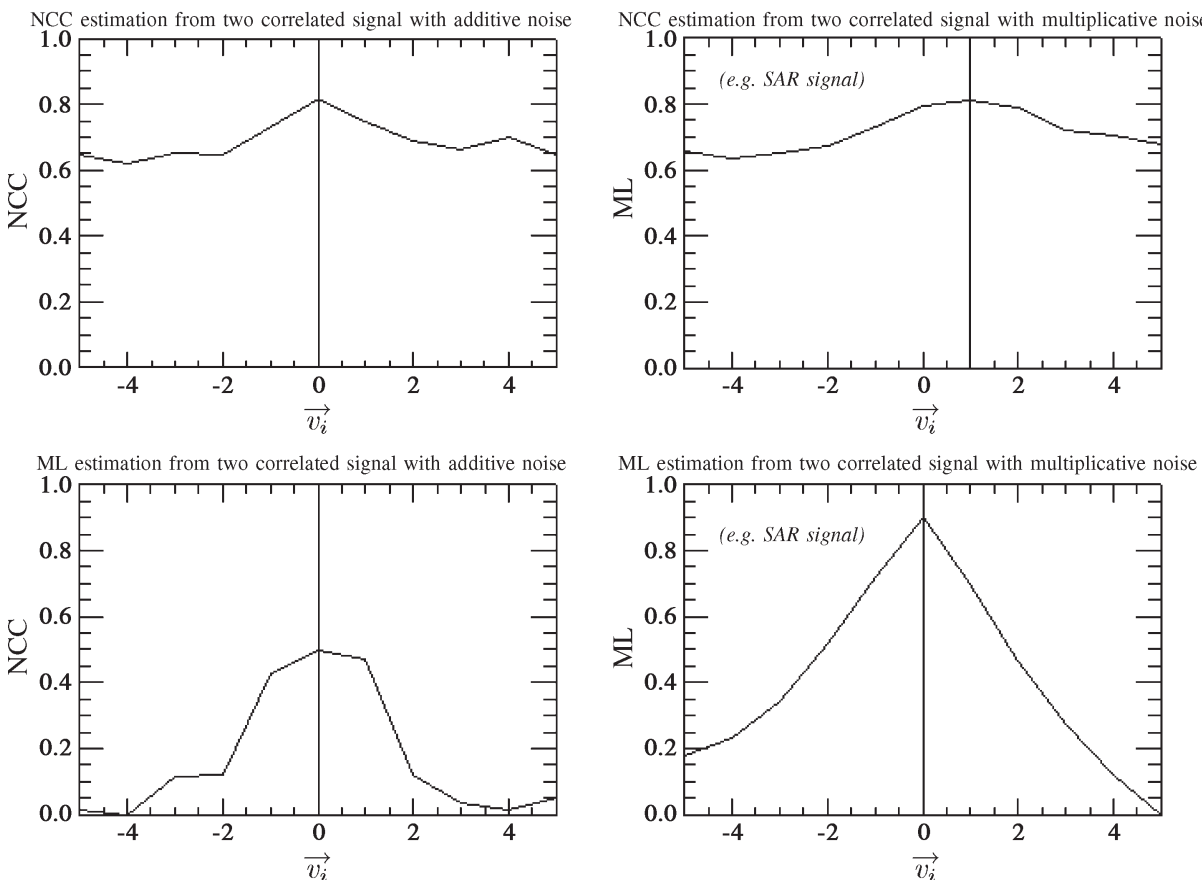

(b)

Fig. 2. (a) 1-D time-domain signals with additive and multiplicative noise complements. (b) Displacement estimations using the NCC and the ML techniques.

TABLE I

ENVISAT ACQUISITIONS OVER INYLTSHIK GLACIER

\begin{tabular}{|c|c|c|c|}
\hline $\begin{array}{c}\text { Temporal } \\
\text { pair }\end{array}$ & Baseline & $\begin{array}{l}\text { Temporal } \\
\text { resolution }\end{array}$ & $\begin{array}{c}\text { System } \\
\text { Parameters (Descending) }\end{array}$ \\
\hline 1 Aug.-Sep. & $360.40 \mathrm{~m}$ & 35 days & Range pixel spacing $4.0 \mathrm{~m}$ \\
\hline 2 Aug.-Oct. & $783.54 \mathrm{~m}$ & 70 days & Azimuth pixel spacing $7.8 \mathrm{~m}$ \\
\hline 3 Sep.-Oct. & $1124.10 \mathrm{~m}$ & 35 days & Offnadir angle $22^{\circ} .65$ \\
\hline
\end{tabular}

image sequences, can be used. On the same area, the wellknown texture tracking technique known as NCC was also employed to better understand the behavior and the gain of the ML texture tracking technique. Considering the loss of coherence over the glacier [Fig. 3(a)], it is obvious that the proposed technique is compared to the incoherent NCC technique and not with the coherent one. Fig. 3(b) shows a typical DInSAR result for a fast-moving glacier (no reliable phase information throughout the glacier).

For both the ML and the NCC motion estimation algorithms, the similarity measurement is built by shifting a small sample window from one image in a search window from another image and by calculating the similarity for each window. To 


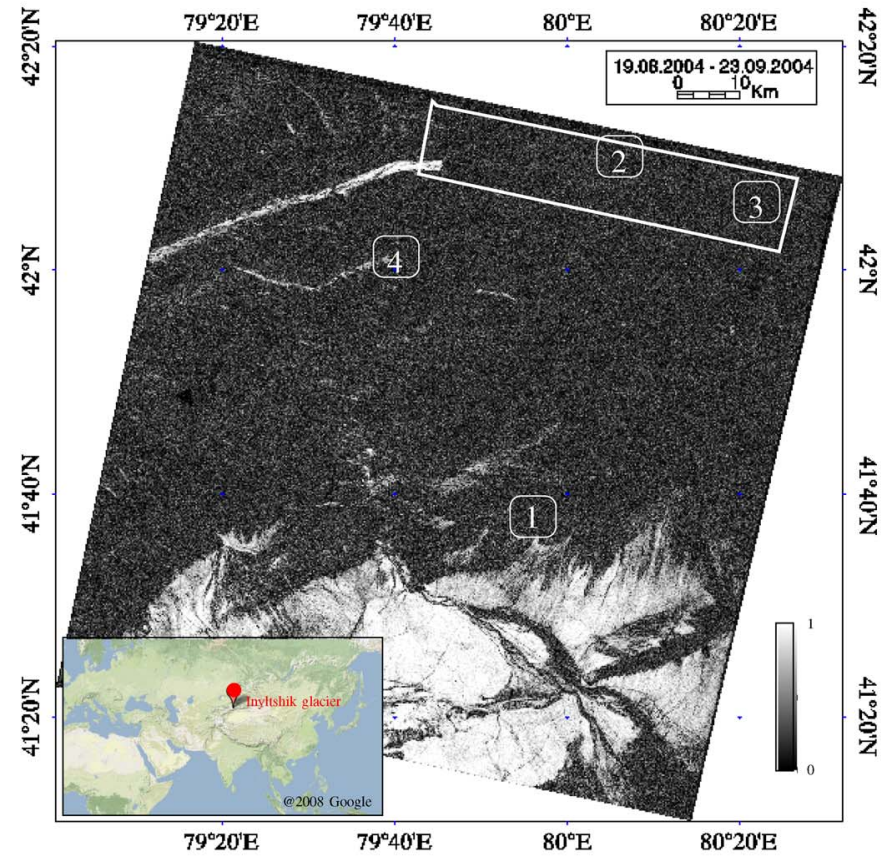

(a)

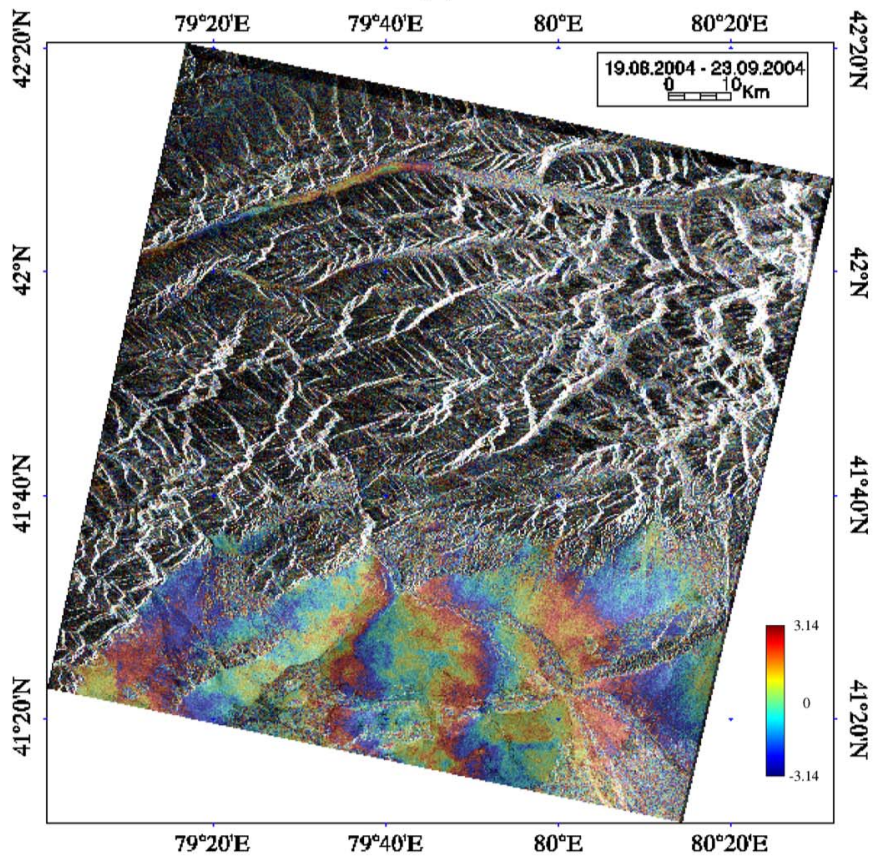

(b)

Fig. 3. (a) DInSAR coherence image. The numbered areas plotted over the coherence image are test areas shown in Fig. 4, and the white rectangle indicates the location of Inyltshik glacier. (b) LOS surface displacement map of the test area, derived from ENVISAT SAR data using DInSAR technique. The intensity represents the radar signal, and a color cycle corresponds to DInSAR phase. Interferometric results were obtained from the I.D.I.O.T. (InSAR Deformation Inspection and Observation Tool) software, developed by the Computer Vision and Remote Sensing Group of the Berlin University of Technology (see, e.g., [20] and [21]).

increase computational efficiency, the shifting procedure was performed in the frequency domain by fast Fourier transform. As indicated by de Lange et al. [14], the block size having a ratio of $1 / 4$ (i.e., approximately square area on the ground) between range and azimuth direction gives the best performances
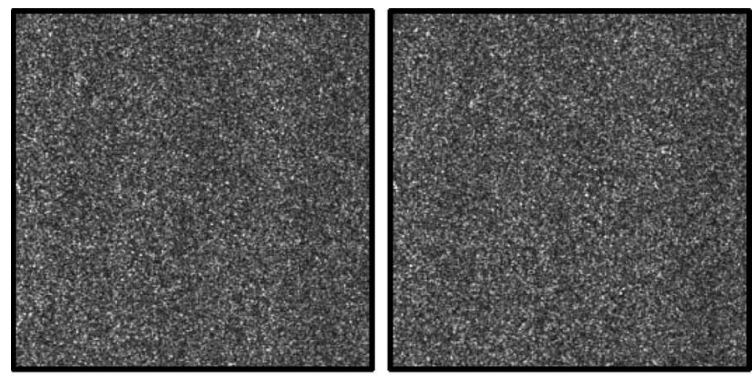

(a)
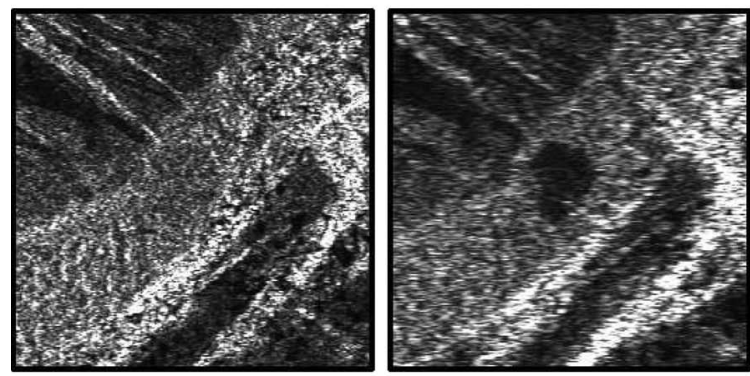

(b)
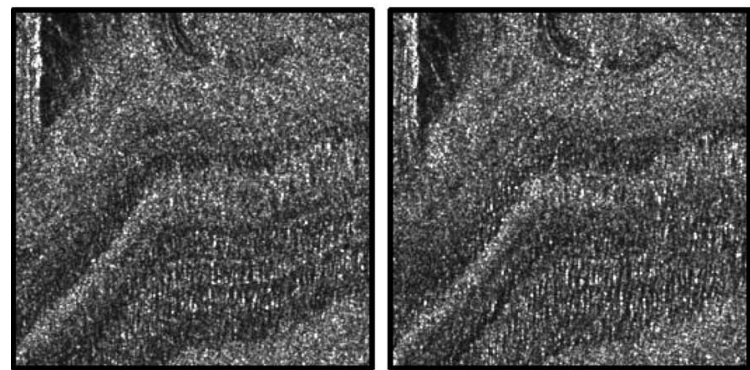

(c)
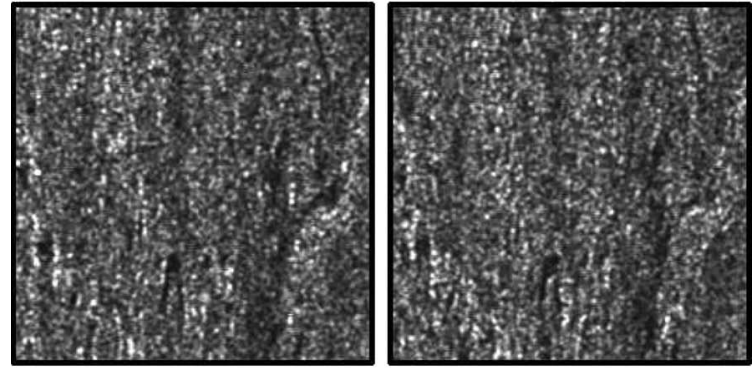

(d)

Fig. 4. (a) Intensity images of no-motion area. (b) Intensity images of lake ice. Merzbacher Lake can easily be recognized in September image based on its low returns; however, the lake is almost invisible in August image based on its high returns. The different characteristic of radar responses is related to ice thickness during the acquisition time. (c) Typical glacier reflectance on SAR images. (d) Intensity images of the small part of the glacier that has a coherent characteristic. In all chosen area, the first subimage is extracted from ENVISAT image acquired at 19th of August, and the second is from ENVISAT image acquired at 23rd of September.

for matching algorithms. Here, the influence of the sample block size on texture tracking was tested with blocks having a size of $16 \times 64,32 \times 128$, and $64 \times 256$ pixels.

Experimental results on real data are divided into two parts. The first part includes the performance analysis of NCC and ML motion estimators in specific areas. The second part shows the performance analysis and the velocity maps obtained from the entire glacier. 


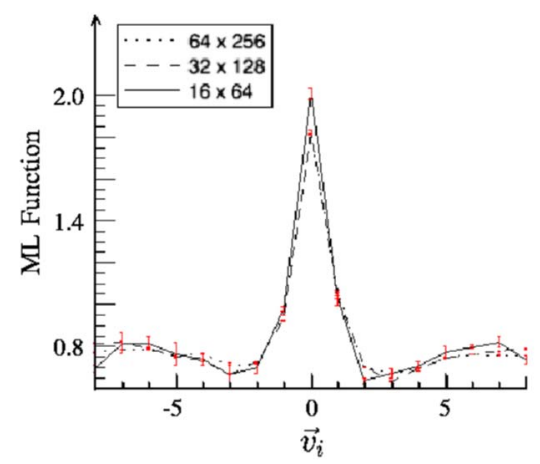

(a)

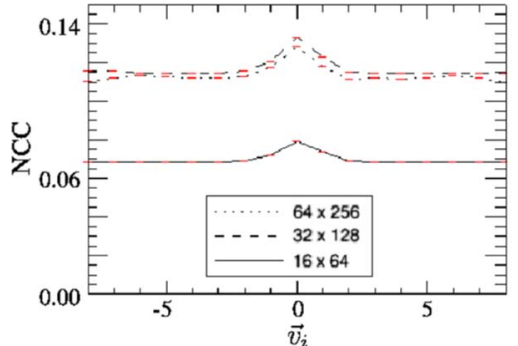

(b)

Fig. 5. (a) Averaged ML and (b) NCC estimators considering no-motion area at azimuth direction. The error bars were constructed with the dimension of $200 \times 200$ pixels.

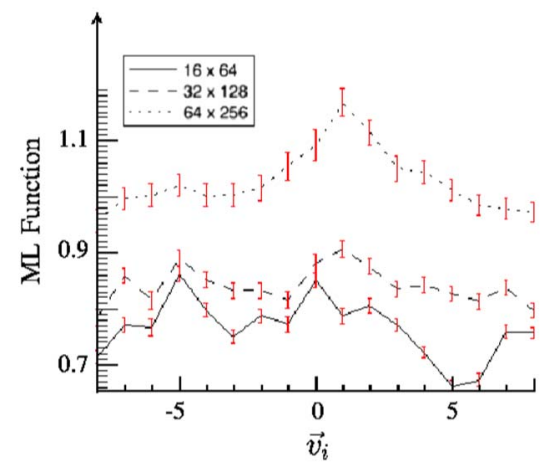

(a)

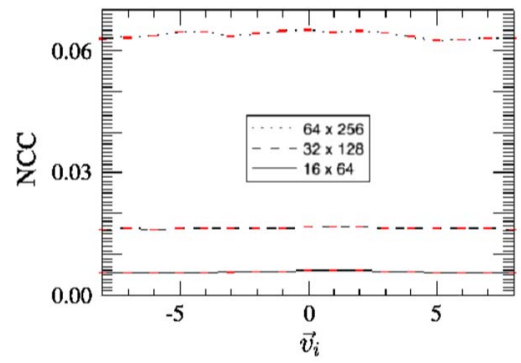

(b)

Fig. 6. (a) Averaged ML and (b) NCC estimators considering land-use boundary-change-based motion. The error bars were constructed from approximately the whole lake through azimuth direction.

\section{A. Motion Estimation at Specific Areas}

There are two different possibilities for performance evaluation of motion estimators on real data. The first is testing the performance of both estimations in an area without motion, where the correct motion $\left(\vec{v}_{i}=0\right)$ is known. In the second case, the motion is not known and the evaluation can be performed based on the distribution of estimators (the behavior of estimators through candidate shifts).

The ML and NCC estimators were implemented in the selected areas, both with block sizes of $16 \times 64,32 \times 128$, and $64 \times 256$. For each area, the distribution of estimators was calculated for different candidate shifts between two images. The distributions were generated by averaging the ML and NCC estimators from the selected area. Error bars, i.e., total standard deviations away from the mean values, were used to show distributions' uncertainty. The resulting distributions from both techniques were compared to each other.

No Motion: First, the proposed and the NCC approaches were tested in the no-motion area [Fig. 4(a)], extracted from glacier data sets. Exploiting the well-known NCC and the ML motion estimation approaches on that area resulted in the characteristic functions shown in Fig. 5. As expected, both approaches result in a maximum at $\vec{v}_{i}=0$. However, the confidence measure (the relative ratio of likelihood values) of the estimation is much better for the ML estimation than for the NCC. Homogeneous intensity supplies little information for matching algorithms, which can be more critical in NCC due to its numerical subtraction procedure. In contrast, the ML function considers the intensity ratio. Although increasing block size has not provided similar results for both techniques, the characteristics of the error bars are almost the same for both techniques: A larger block size results in a higher standard deviation.

Land-Use Boundary-Change-Based Motion: The physical properties of the imaged objects may change over time in a natural manner. Thus, the problem, which is to match objects that have inherent variations over time, can appear. A typical example of this kind of inherent changes regarding the glacial environment is lake ice [Fig. 4(b)]. In practice, it results in a mismatch based on the variation of the backscattering of objects. Fig. 6 shows the ML and NCC matching estimators at Merzbacher Lake area. In that area, both techniques have a different kind of distribution. The ML motion estimation approach supplies a satisfactory estimation. On the other hand, the NCC approach has approximately uniform behavior through shift vectors. It should be noted that small NCC values (no desirable peak) and small amount of error bars can be related to an inherent physical variation of the selected areas during the time period. ${ }^{3}$

\footnotetext{
${ }^{3}$ The distribution of matching algorithms can also be used in change detection analysis. It should be remembered that change detection problems are also time- and scale-dependent problems [22].
} 


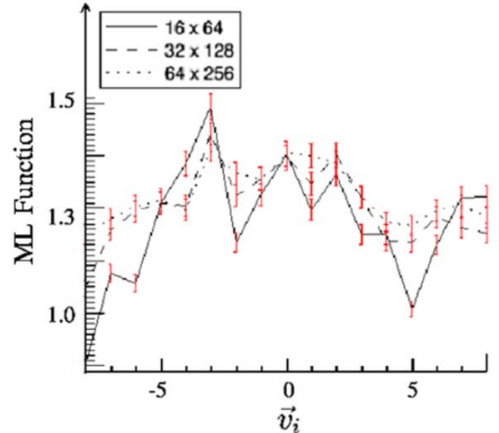

(a)

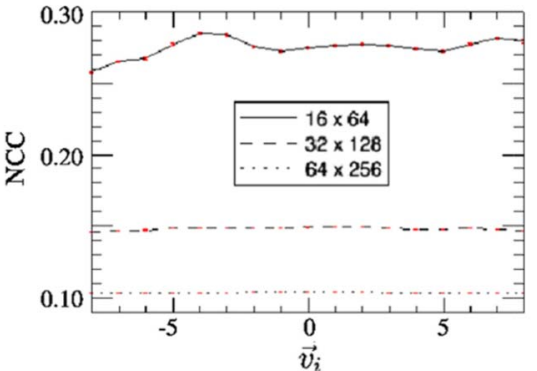

(b)

Fig. 7. (a) Averaged ML and (b) NCC estimators considering typical glacier motion in azimuth direction. The error bars were constructed with the dimension of $100 \times 100$ pixels.

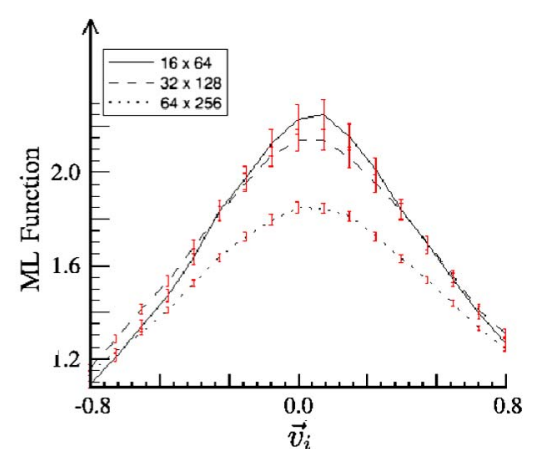

(a)

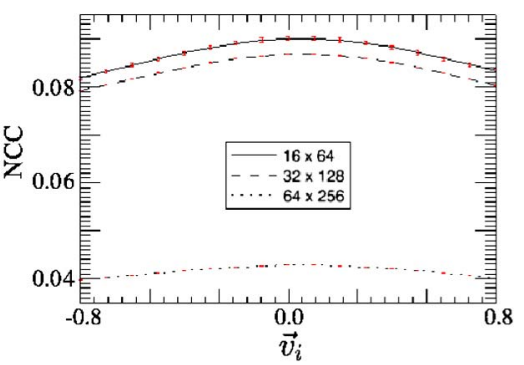

(b)

Fig. 8. (a) Averaged ML and (b) NCC estimators extracted from the correlated part of glacier $(0 \leq \rho \leq 0.4)$. The error bars were constructed with the dimension of $100 \times 100$ pixels.

Offset Motion: This is a common (with respect to computer vision as well as remote sensing applications) motion type, which occurs frequently in glacierized areas. Although land-use boundary-change-based motion can seldom be analyzed with coherent data, offset motion is more likely to result in coherent data. Therefore, this motion type was analyzed for two different areas shown in Fig. 4(c) and (d). According to Fig. 7, the comparison of NCC and ML motion matching allows the complete surface flow measurement: Both approaches supply the same shifts at a small scale. However, larger scale estimation, particularly for NCC approach, can cause a mismatch in such kind of spatial/temporal characteristics. This example also reveals that a larger scale does not always supply a statistically highly accurate estimation. It should be remembered that every estimation is directly related to the characteristics of the samples, and not only to the number of samples. Fig. 8 shows an average of the ML and NCC motion estimation from a coherent area [Fig. 4(d)]. In this case, the images were oversampled by a factor of ten to detect smaller offsets. As inferred from Fig. 8, both approaches have a similar, approximately symmetric, distribution around $\vec{v}_{i}=0$.

From the analysis of four experimental results, it is found that the variation of the ML estimator through scale is not as large as the one of the NCC estimator. It may be concluded that using a multiplicative speckle model results in an improvement of the matching performance.
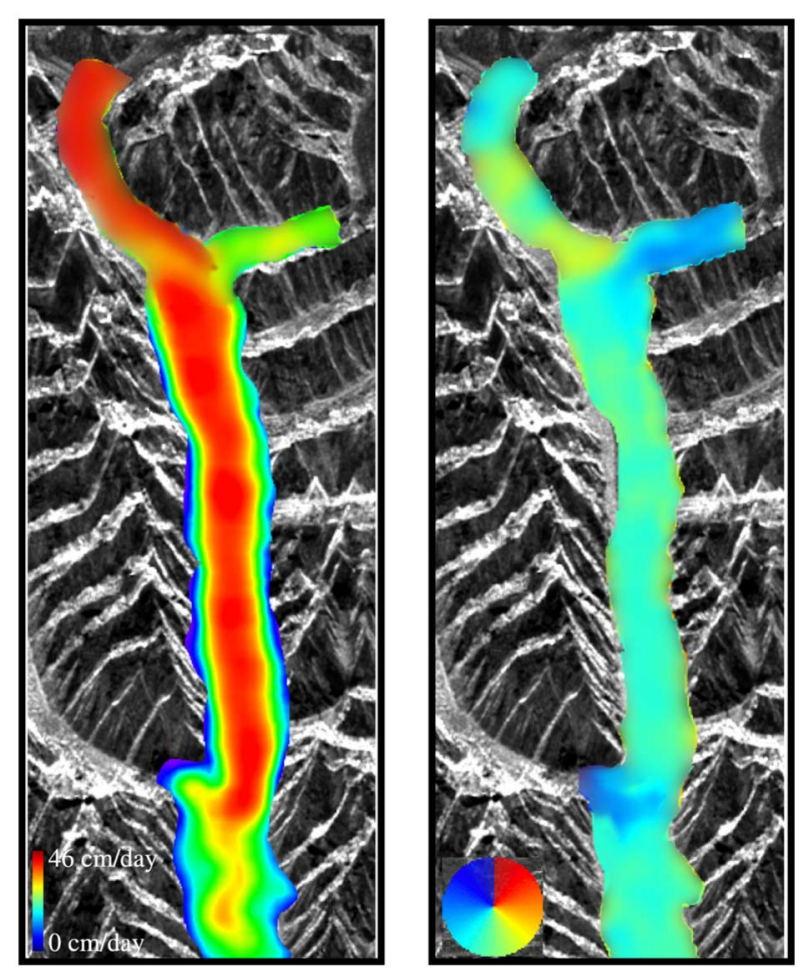

Fig. 9. (a) Velocity map produced using ML motion estimator. Velocity fields are determined from the division of the shift (displacement) vector field by the time interval between image acquisitions. (b) Direction map of velocity vectors. 


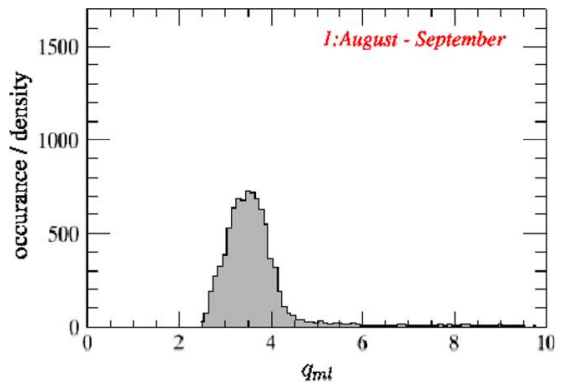

(a)

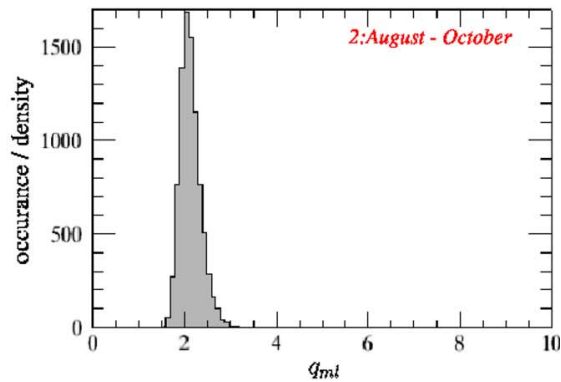

(b)

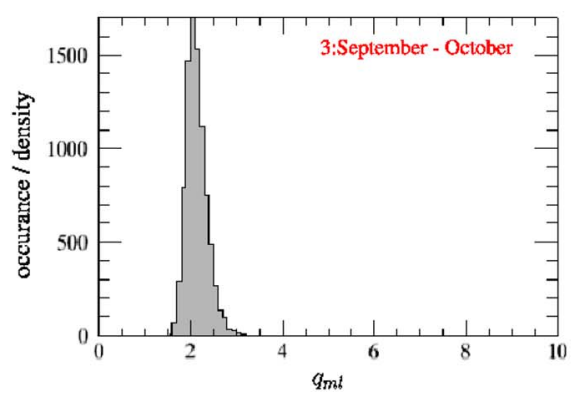

(c)

Fig. 10. Normalized quality assessments (17) of velocity measurements obtained through summer 2004 with ENVISAT images.

\section{B. Velocity Analysis for the Whole Glacier}

This section contains a concise presentation of the ML estimator. It is not intended to give an in-depth geophysical analysis but merely an example of motion estimation using the ML technique. The proposed ML texture tracking technique was employed over the Inyltshik glacier and used to estimate the velocity of the glacier where the application of differential interferometry was limited by phase noise. The velocity and its direction maps, obtained from a window of $128 \times$ 512 pixels, are shown in Fig. 9. The velocity map from the ML estimation shows the typical surface velocity pattern of glaciers with the highest velocities in the center of the glacier and a strong gradient toward the boundary. Note that in a portion of the glacier in the bottom of Fig. 9(b), the direction of the surface velocity is toward upward direction, which would not be expected. This happens due to the presence of the Lake Merzbacher in the left-hand side of the glacier, and the draining of the glacier into the lake. A more detailed study about the geophysical properties of the surface velocity of the Inyltshik glacier can be found in [23].

To test the performance of the estimation, the histogram of the following ratio was calculated:

$$
q_{m l}=\frac{\max (m l)-\operatorname{mean}(m l)}{\operatorname{mean}(m l)-\min (m l)}
$$

where $q_{m l}$ and $m l$ are replaced by $q_{\mathrm{NCC}}$ and $n c c$ for NCC estimation and $q_{m l} / q_{n c c}$ indicates the quality of $m l / n c c$ estimators for different shifts. Fig. 10 shows histograms of the normalized quality assessments of velocity measurements obtained through the images of summer 2004. From the quality analysis (Fig. 10), it is found that the velocity measurement extracted from August-September pair has a higher confidence and that there is a slight difference between the quality of measurements

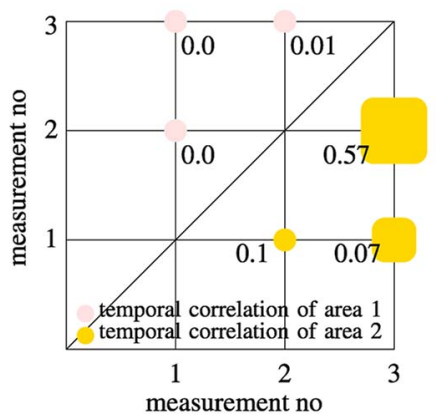

Fig. 11. Correlation coefficients, within the variations of the velocity measurement based on different temporal resolutions, were calculated for the selected two areas. Here, 1 is the result of the ML estimator with a time interval of August-September, 2 is the one of August-October, and 3 is the one of September-October.

obtained from August-October and September-October pairs. The last experiment (Fig. 11) shows the correlation of the measurements that were estimated for different time periods. In other words, the correlation coefficient is used to see how well all measurements vary jointly. The correlation analysis was performed over two different areas. In the first area, measurement 1 was obtained from a data set having a correlated speckle pattern, while measurement 2 and measurement 3 were obtained from a data set characterized by an independent speckle pattern. In the second area, measurements 1,2 , and 3 were all obtained from data sets having uncorrelated speckle patterns. The correlation coefficients between measurements for both areas were calculated. It can be seen from Fig. 11 that for the first area, there is no correlation between measurements, meaning that a large variation in velocity exists over the time period. However, for the second area, the measurements 2 and 3 maintain a higher correlation (which means that there is an approximately uniform motion through these time periods) than the others. 


\section{CONCLUSION AND Discussion}

The ENVISAT-ASAR Inyltshik glacier data set has been processed to monitor glacier surface velocity in the context of multitemporal SAR image interpretation. A new algorithm for intensity matching, which is based on the assumption that SAR images are characterized by a multiplicative speckle model, has been introduced and applied. The proposed algorithm uses ML decision rules evaluated from the density function of the ratio of two speckle patterns. Even though a new intensity-based matching algorithm is proposed, particularly for incoherent data sets, the analysis of the proposed technique was also performed for correlated data sets. The bivariate gamma distribution with gamma marginals has been used for the analysis of correlated speckle behavior. It has been shown that the use of optimized parameters (ML) can improve the classical intensity matching algorithm without any assumption about the existence of correlation and/or decorrelation. The proposed estimator has been compared to the classical incoherent NCC estimator and has been shown to have a more robust behavior than the incoherent NCC estimator, particularly in areas including variation in reflectance over the time period.

Based on the presented results and experiences, the effectiveness of matching algorithms is related to the presence of identical features among SAR images at the scale defined by blocks. Here, scale can be thought of as a geographic meaning (working block). In every remote sensing application, the main problem is that there is a tradeoff between scale and accuracy: Larger scale (big block size) causes higher statistical accuracy but lower resolution; and lower scale causes more detailed information but lower statistical accuracy. The estimation performance of the ML velocity estimator may be improved by increasing the scale. However, the scale must be commensurate with the size of working block including homogeneous displacement; otherwise, the velocity estimation is affected by differing displacement vectors. Generally, a multiscale structure can be helpful in solving the block size problem. Although multiscale approaches increase the accuracy [22], [24], the development of an optimal approach may be application- and area-dependent. Related experiences show that a multiscale approach should be based on shape, texture, and land-use variation, taking temporal resolution into account, and is an area of future research in tracking techniques.

The main results of the proposed approach are that using SAR statistical characteristics improves the quality of the matching algorithms and that matching and change detection algorithms are complementary to each other. The quality of matching algorithms can be increased by considering the sensitivity to detect the change statistics. In addition, encouraged by these results, this single-channel SAR system work will be implemented to multichannel (e.g., polarimetric data) SAR systems using its multiplicative plus additive noise model.

It has already been shown that with the relatively low spatial resolution of ENVISAT, the achieved velocity accuracy is sufficient for monitoring moderately fast glacier flows. Recent developments in SAR technologies supply much higher spatial resolution, which will further enhance the precision of the proposed approach. The TerraSAR-X and RADARSAT-2 sensors will be interesting options for the detection and analysis of much slower ground movement. In addition, TerraSAR-X works at X-band (ENVISAT and ERS have C-band) which may result in incoherent data sets in the case of the monitoring of alpine glaciers. Notwithstanding its sparseness, sometimes, coherent data sets may be available. In such a case, the correlation can be easily included in the ML estimator, and this implementation allows that the proposed approach can be operational in the presence of either correlated or uncorrelated data sets. Thus, the development of monitoring techniques using amplitude data can be interesting for future high-resolution SAR systems, and it can provide complementary information for interferometric techniques (in the case of the presence of coherent data).

\section{APPENDIX I}

\section{A. ML Motion Estimation for Logarithmic Images}

Taking the natural logarithm of (5) with $\gamma=1$ leads to $x_{i j}=$ $\alpha_{i j}+y_{i j}$. If $\underline{\alpha}=\ln \alpha, p_{\underline{\alpha}}(\underline{\alpha})=p_{\alpha}(\underline{\alpha}) /(1 / \alpha)$, where $\overline{p_{\alpha}}(\alpha)$ shows the new speckle distribution indicated by (7). Then, (7) is rewritten for logarithmic scale images by substituting $\alpha=\exp (\underline{\alpha})$ in $p_{\underline{\alpha}}(\underline{\alpha})$ as in the following:

$$
p\left(x_{i} \mid y_{i}, \vec{v}_{i}\right)=\prod_{j=1}^{k} \frac{\left(\exp \left(\underline{\alpha_{i j}}\right)\right)^{N}}{\left(1+\exp \left(\underline{\alpha_{i j}}\right)\right)^{2 N}} .
$$

After removing the remaining constant term, the log-likelihood function can be written as follows:

$$
\sum_{j=1}^{k} \underline{x_{i j}}-\underline{y_{i j}}-2 \ln \left(1+\exp \left(\underline{x_{i j}}-\underline{y_{i j}}\right)\right) .
$$

\section{APPENDIX II}

\section{A. Distribution of the Ratio of Two Correlated Speckle Patterns}

If $\eta^{\prime}$ and $\eta^{\prime \prime}$ are governed by a gamma distribution with scale parameter $1 / N$ and they are correlated according to the correlation coefficient $\left(\rho=\operatorname{cov}\left(\eta^{\prime}, \eta^{\prime \prime}\right) / \sqrt{\operatorname{var}\left(\eta^{\prime}\right) \operatorname{var}\left(\eta^{\prime \prime}\right)}\right)(0 \leq \rho \leq 1)$, their joint pdf is given by using the definition of bivariate gamma distribution [18]

$$
\begin{array}{r}
p_{\eta^{\prime \prime} \eta^{\prime}}\left(\eta^{\prime \prime}, \eta^{\prime}\right)=\frac{N^{N+1}\left(\eta^{\prime} \eta^{\prime \prime}\right)^{\frac{N-1}{2}}}{\Gamma(N)(1-\rho)^{\frac{N-1}{2}}} \exp \left(-\frac{N\left(\eta^{\prime}+\eta^{\prime \prime}\right)}{1-\rho}\right) \\
\times I_{N-1}\left(\frac{2 \sqrt{\rho}}{1-\rho} N \sqrt{\eta^{\prime} \eta^{\prime \prime}}\right)
\end{array}
$$

with

$$
I_{N-1}(x)=\sum_{l=0}^{\infty} \frac{\left(\frac{x}{2}\right)^{2 l+N-1}}{l ! \Gamma(l+N)}
$$

where $I_{N-1}$ is the modified Bessel function of the first kind. 
The ratio pdf of $\alpha=\eta^{\prime \prime} / \eta^{\prime}$ is derived by [15, eq. 6.60]

$$
p_{\alpha}(\alpha)=\int_{0}^{\infty} \eta^{\prime} p_{\eta^{\prime \prime} \eta^{\prime}}\left(\alpha \eta^{\prime}, \eta^{\prime}\right) d \eta^{\prime}, \quad \eta^{\prime}, \eta^{\prime \prime} \geq 0 .
$$

Substitution of the joint pdf (B1) into (B3) follows that

$$
\begin{aligned}
p_{\alpha}(\alpha)= & \frac{N^{N+1} \alpha^{\frac{N-1}{2}}}{\Gamma(N)(1-\rho) \rho^{\frac{N-1}{2}}} \sum_{l=0}^{\infty} \frac{\left(\frac{N \sqrt{\rho \alpha}}{1-\rho}\right)^{2 l+N-1}}{l ! \Gamma(l+N)} \\
& \times \int_{0}^{\infty} \eta^{\prime 2 l+2 N-1} \exp \left(-\eta^{\prime} \frac{N(1+\alpha)}{(1-\alpha)}\right) d \eta^{\prime} \\
= & \frac{(\alpha(1-\rho))^{N} 2 N^{-1}}{\alpha(1+\alpha) \Gamma(N)} \sum_{l=0}^{\infty} \frac{\Gamma(2 l+2 N)}{l ! \Gamma(l+N)}\left(\frac{\rho \alpha}{(1+\alpha)^{2}}\right)^{l} \\
= & \frac{\Gamma(2 N)(1-\rho) \alpha^{N}}{\alpha \Gamma(N)^{2}(1+\alpha)^{2 N}} F_{0}\left(\frac{1+2 N}{2} ;-; \frac{4 \rho \alpha}{(\alpha+1)^{2}}\right)
\end{aligned}
$$

where ${ }_{1} F_{0}(a ;-; x)$ is a generalized hypergeometric function which converges for $|x|<1$ as in the present case. The last step of (B4) is performed through Legendre duplication.

\section{B. Moments of Correlated Speckle Ratio}

The $h$ th moment of $\alpha=\eta^{\prime \prime} / \eta^{\prime}$, i.e., $E\left[\alpha^{h}\right]$ is

$$
\begin{aligned}
E\left[\alpha^{h}\right]= & \int_{0}^{\infty} \alpha^{h} p_{\alpha}(\alpha) d \alpha \\
= & \frac{\Gamma(2 N)}{\Gamma(N) \Gamma(N)}(1-\rho)^{N} \int_{0}^{\infty} \frac{\alpha^{N+h-1}}{(1+\alpha)^{2 N}} \\
& \times{ }_{1} F_{0}\left(N+\frac{1}{2} ;-; \frac{4 \rho \alpha}{(\alpha+1)^{2}}\right) d \alpha \\
= & \frac{\Gamma(2 N)}{\Gamma(N) \Gamma(N)}(1-\rho)^{N} \sum_{l=0}^{\infty} \frac{\left(N+\frac{1}{2}\right)_{l}(4 \rho)^{l}}{l !} \\
& \times \int_{0}^{\infty} \frac{\alpha^{h+l+N-1}}{(\alpha+1)^{2 l+2 N}} d \alpha .
\end{aligned}
$$

With Euler Beta function $(B(a, b)=\Gamma(a) \Gamma(b) / \Gamma(a+b), a \geq$ $0, b \geq 0)$ and Pochhammer symbols $\left((a)_{l}=a(a+1)(a+\right.$ $2), \ldots,(a+l-1), l=1,2,3, \ldots)$, (B5) can be written in a simple closed form as in the following:

$$
E\left[\alpha^{h}\right]=\frac{\Gamma(N+h) \Gamma(N-h)}{\Gamma(N)^{2}(1-\rho)^{-N}}{ }_{2} F_{1}(N+h, N-h ; N ; \rho)
$$

where the definition of the hypergeometric function ${ }_{2} F_{1}(a, b$; $c ; z)=\sum_{l=0}^{\infty}\left((a)_{l}(b)_{l} /(c)_{l}\right)\left(z^{l} / l !\right)$ is used. From (B6), it is possible to compute central moments and functions of them,

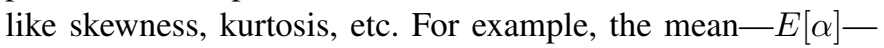

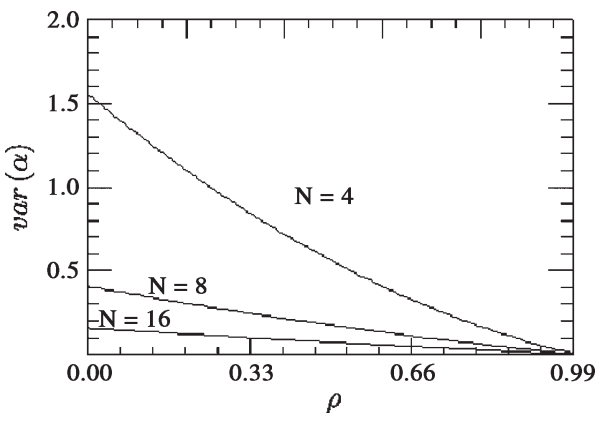

Fig. 12. Behavior of variance of the ratio of two speckle variables as a function of correlation $0 \leq \rho \leq 1$.

and the variance (Fig. 12)—E[ $\left.\alpha^{2}\right]-E[\alpha]^{2}$ —based on $\rho$ are given by

$$
\begin{aligned}
& \rho=0 \rightarrow\left\{\begin{array}{l}
E[\alpha] \quad=\frac{N}{N-1} \\
\operatorname{var}[\alpha]=\frac{N(2 N-1)}{(N-1)^{2}(N-2)}
\end{array}\right. \\
& 0 \leq \rho \leq 1 \rightarrow \begin{cases}E[\alpha]=\frac{N-\rho}{N-1} \\
\operatorname{var}[\alpha]=\frac{N(N+1)}{(N-1)(N-2)} \\
\times\left(1-\frac{4 \rho}{N}+\frac{6 \rho^{2}}{N(N+1)}\right)-\frac{(N-\rho)^{2}}{(N-1)^{2}}\end{cases}
\end{aligned}
$$

where the transform of ${ }_{2} F_{1}(a, b ; c ; x)=(1-x)^{c-a-b}{ }_{2} F_{1}(c-a$, $c-b ; c ; x)$ is used. As a consequence, the new speckle distribution, which is defined as a ratio of two speckle patterns, only depends on their correlation. On the other hand, the probability of an error in matching algorithm increases with aberration on the assumption of $\gamma=\sigma^{\prime \prime} / \sigma^{\prime}=1$. Related to this, it is preferred to work with normalized intensity, even if it is in logarithmic scale.

\section{ACKNOWLEDGMENT}

The authors would like to thank R. Zandoná-Schneider and the anonymous reviewers for their constructive comments for improving this paper and Deutsches GeoForschungsZentrum (GFZ) in Potsdam for providing the ENVISAT data sets of Inyltshik glacier region.

\section{REFERENCES}

[1] Q. X. Wu, S. J. McNeill, and D. Pairman, "Correlation and relaxation labelling: An experimental investigation on fast algorithms," Int. J. Remote Sens., vol. 18, no. 3, pp. 651-662, Feb. 1997.

[2] T. Strozzi, A. Luckman, T. Murray, U. Wegmuller, and C. Werner, "Glacier motion estimation using SAR offset-tracking procedures," IEEE Trans. Geosci. Remote Sens., vol. 40, no. 11, pp. 2384-2391, Nov. 2002.

[3] F. W. Leberl and K. Maurice, "Automated radar image matching experiment," ISPRS J. Photogramm. Remote Sens., vol. 49, no. 3, pp. 19-33, Jun. 1994.

[4] A. N. Evans, "Glacier surface motion computation from digital image sequences," IEEE Trans. Geosci. Remote Sens., vol. 38, no. 2, pp. 1064 1072, Mar. 2000.

[5] K. Chaudhury, R. Mehrotra, and C. Srinivasan, "Detecting 3D flow," in Proc. IEEE Int. Conf. Robot. Autom., San Diego, CA, May 1994, pp. 1073-1078.

[6] R. Kwok, M. Fahnestock, and C. Chan, "Ice sheet motion and topography from radar interferometry," IEEE Trans. Geosci. Remote Sens., vol. 34, no. 1, pp. 189-220, Jan. 1994. 
[7] I. R. Joughin, R. Kwok, and M. A. Fahnestock, "Interferometric estimation of three-dimensional ice-flow using ascending and descending passes," IEEE Trans. Geosci. Remote Sens., vol. 36, no. 1, pp. 25-37, Jan. 1998.

[8] A. Reigber, P. Prats, R. Scheiber, C. Andres, E. Erten, and O. Hellwich, Vermessung der Fliegeschwindigkeit Alpiner Gletscher mit Flugzeuggesttzter Differentieller sar Interferometrie Allgemeine Vermessungs-Nachrichten (AVN), Jul. 2008.

[9] E. Erten, A. Reigber, and O. Hellwich, "Generation of three-dimensional deformation maps from InSAR data using spectral diversity techniques," ISPRS J. Photogramm. Remote Sens. to be published.

[10] R. Scheiber and A. Moreira, "Coregistration of interferometric SAR images using spectral diversity," IEEE Trans. Geosci. Remote Sens., vol. 38, no. 5, pp. 2179-2191, Sep. 2000.

[11] R. Bamler and M. Eineder, "Accuracy of differential shift estimation by correlation and split-bandwidth interferometry for wideband and delta-k SAR systems," IEEE Geosci. Remote Sens. Lett., vol. 2, no. 2, pp. 151155, Apr. 2005.

[12] M. G. Strintzis and I. Kokkinidis, "Maximum likelihood motion estimation in ultrasound image sequences," IEEE Signal Process. Lett., vol. 4, no. 6, pp. 156-157, Jun. 1997.

[13] R. Bamler, "Interferometric stereo radargrammetry: Absolute height determination from ERS-ENVISAT interferograms," in Proc. Int. Geosci. Remote Sens. Symp., Honolulu, HI, Jul. 2000, vol. 2, pp. 980-984.

[14] R. de Lange, A. Luckman, and T. Murray, "Improvement of satellite radar feature tracking for ice velocity derivation by spatial frequency filtering," IEEE Trans. Geosci. Remote Sens., vol. 45, no. 7, pp. 2309-2318, Jul. 2007.

[15] A. Papoulis, Probability, Random Variables, and Stochastic Processes, 4th ed. Singapore: McGraw-Hill, 2002.

[16] C. Oliver and S. Quegan, Understanding Synthetic Aperture Radar Images. Norwood, MA: Artech House, 1998.

[17] R. Touzi, A. Lopes, and P. Bousquet, "A statistical and geometrical edge detector for SAR images," IEEE Trans. Geosci. Remote Sens., vol. 26, no. 6, pp. 764-773, Nov. 1988.

[18] H. Holm and M. S. Alouini, "Sum and difference of two squared correlated Nakagami variates in connection with the Mckay distribution," IEEE Trans. Commun., vol. 52, no. 8, pp. 1367-1376, Aug. 2004.

[19] F. Chatelain, J. Y. Tourneret, J. Inglada, and A. Ferrari, "Bivariate gamma distributions for image registration and change detection," IEEE Trans. Image Process., vol. 16, no. 7, pp. 1796-1806, Jul. 2007.

[20] A. Reigber, E. Erten, S. Guillaso, and O. Hellwich, "Idiot: A free and easy-to-use software tool for DInSAR analysis," in Proc. Envisat Symp., Montreux, Switzerland, Apr. 2007, pp. 23-27.

[21] I.D.I.O.T. Homepage. [Online]. Available: http://www.cv.tu-berlin.de/ idiot/

[22] F. Bovolo and L. Bruzzone, "A detail-preserving scale-driven approach to change detection in multitemporal SAR images," IEEE Trans. Geosci. Remote Sens., vol. 43, no. 12, pp. 2963-2972, Dec. 2005.

[23] C. Mayer, W. Hagg, A. Lambrecht, A. Helm, and K. Scharrer, "Postdrainage ice dam response at Lake Merzbacher, Inylchek glacier, Kyrgyzstan,” Geografiska Annaler, vol. 90, no. 1, pp. 87-96, Mar. 2008.

[24] J. Inglada and G. Mercier, "A new statistical similarity measure for change detection in multitemporal SAR images and its extension to multiscale change analysis," IEEE Trans. Geosci. Remote Sens., vol. 45, no. 5, pp. 1432-1445, May 2007.

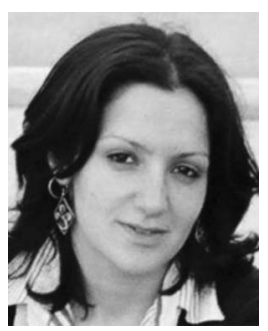

Esra Erten (S'06) was born in Rize, Turkey, in 1980. She received the B.S. degree in geodesy and photogrammetry engineering and the M.S.E.E. degree in satellite communication and remote sensing from Istanbul Technical University, Istanbul, Turkey, in 2003 and 2005, respectively. She is currently working toward the Ph.D. degree in the Department of Computer Vision and Remote Sensing, Berlin University of Technology, Berlin, Germany, in collaboration with the Microwaves and Radar Institute, German Aerospace Center (DLR),

Oberpfaffenhofen, Germany.

Her research interests include information extraction and image understanding from SAR and optical images, in particular information theory, SAR polarimetry, and interferometry.

Ms. Erten was awarded, during her bachelor degree, a 2004 Best Student Paper Award at XXth International Society of Photogrammetry and Remote Sensing Congress, with the topic of Forest Fire Risk Mapping from Satellite Imagery and GIS a Case Study.

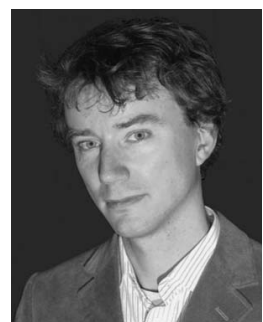

Andreas Reigber (M'02) was born in Munich, Germany, in 1970. He received the Diploma degree in physics from the University of Konstanz, Konstanz, Germany, in 1997, the Ph.D. degree from the University of Stuttgart, Stuttgart, Germany, in 2001, and the habilitation from the Berlin University of Technology, Berlin, Germany, in 2008

From 1996 to 2000, he was with the Microwave and Radar Institute, German Aerospace Center (DLR), Oberpfaffenhofen, Germany, working in the field of polarimetric SAR tomography. In 2001, he joined the Antenna, Radar and Telecom Laboratories, University of Rennes 1 , Rennes, France, for a postdoc on radar polarimetry and polarimetric interferometry. From 2002 to 2007, he was a Research Associate with the Computer Vision and Remote Sensing Laboratories, Berlin University of Technology. Since 2008, he has been with the DLR Microwave and Radar Institute, where he is currently the Head of the SAR Technology Department. His current main research interests include the various aspects of multimodal SAR, like SAR interferometry, SAR polarimetry, SAR tomography, and time-frequency analyses, and also hyperspectral remote sensing and the application of computer vision and machine learning approaches in remote sensing.

Dr. Reigber has received the EUSAR 2000 Student Prize Paper Award for an article on SAR remote sensing of forests, the IEEE TGRS Transactions Prize Paper Award in 2001 for a work on polarimetric SAR tomography, and the IEEE TGRS Letters Prize Paper Award in 2006 for a work on multipass SAR processing. He also coauthored three papers which have been successful at the student paper competitions of IGARSS'05 (first prize), EUSAR'06 (third prize), and IGARSS'07 (second prize).

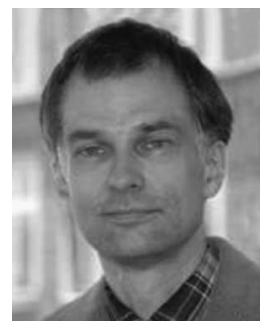

Olaf Hellwich (SM'06) was born in 1962. He received the degree in surveying engineering from the University of New Brunswick, Fredericton, NB, Canada, and the Ph.D. degree from Technische Universität München, Munich, Germany, in 1997.

He then headed the Remote Sensing Group of Technische Universität München. Since 2001, he has been a Professor with Technische Universität Berlin (TUB), Berlin, Germany, initially with the Department of Photogrammetry and Cartography and, since 2004, with the Department of Computer Vision and Remote Sensing. He is also currently the Dean of TUB's Faculty for Electrical Engineering and Computer Science. His research interests include 3-D object reconstruction, e.g., trinocular video sequences; object recognition, e.g. realtime head pose estimation; and synthetic aperture radar remote sensing, e.g. for surface motion estimation.

Prof. Hellwich was the recipient of the Hansa Luftbild Price of the German Society for Photogrammetry and Remote Sensing in 2000.

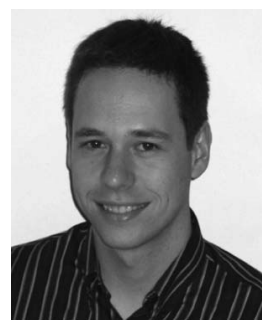

Pau Prats (S'03-M'06) was born in Madrid, Spain, in 1977. He received the Ingeniero degree in telecommunication engineering and the Ph.D. degree from the Universitat Politècnica de Catalunya (UPC), Barcelona, Spain, in 2001 and 2006, respectively.

In 2001, he was a Research Assistant with the Institute of Geomatics, Spain, designing a subaperture SAR processor. In 2002, he was with the Department of Signal Theory and Communications, UPC, working in the field of airborne repeat-pass interferometry and airborne differential SAR interferometry. From December 2002 to August 2006, he was an Assistant Professor with the Department of Telecommunications and Systems Engineering, Universitat Autònoma de Barcelona, Spain. Since August 2006, he has been a Research Scientist with the SAR Processing Group, Microwave and Radar Institute, German Aerospace Center, Oberpfaffenhofen, Germany, working in the field of SAR processing and interferometry. His research interests include SAR processing, airborne motion compensation, single/repeat-pass interferometry, and differential interferometry with airborne systems.

Dr. Prats was a recipient of the First Prize of the Student Paper Competition at IGARSS 2005 Conference, held in Seoul, Korea. 